\title{
Mass, Fine Structure Constant, and the Classification of Elementary Particles by Masses
}

\author{
Khachatur Kirakosyan \\ Institute of Chemical Physics, Armenian National Academy of Sciences, Erevan, Armenia \\ Email:khkirakosyan@gmail.com
}

How to cite this paper: Kirakosyan, K. (2021) Mass, Fine Structure Constant, and the Classification of Elementary Particles by Masses. Journal of Modern Physics, 12, 988-1004.

https://doi.org/10.4236/jmp.2021.127061

Received: April 9, 2021

Accepted: May 22, 2021

Published: May 25, 2021

Copyright $\odot 2021$ by author(s) and Scientific Research Publishing Inc. This work is licensed under the Creative Commons Attribution International License (CC BY 4.0).

http://creativecommons.org/licenses/by/4.0/

\section{(c) (i) Open Access}

\begin{abstract}
The equations of motion of physical bodies are given, the characteristic parameters of which become the basis for determining a fundamental property of all matter- "mass". The equations of motion are characterized by two constants, the derivative of one of which is the fine structure constant. Using these constants, energy scales are compiled, which are the basis for classifying particles by mass.
\end{abstract}

\section{Keywords}

Mass, Energy, Structure, Quantum Numbers, Elementary Particles

\section{Introduction}

In modern theoretical views, the origin of the mass and the fine structure constant (the symbol $\alpha$ ) one relates with the phenomena of interaction [1]-[6], thus the search for a connection between the mass of elementary particles (EP) and $\alpha$ seems to be quite natural. However, the establishment of this connection is complicated by the fact that the contents of $\alpha$ and mass are not sufficiently revealed. Regarding the content of $\alpha$, Feynman remarks very nicely: "one of the greatest damn mysteries of physics: a magic number that comes to us with no understanding by man" [7]. In practice, the conclusion given in [8] [9] that the concept of mass is shrouded in serious uncertainties and is one of the most important unsolved problems of modern physics remains relevant.

In the proposed work, based on the results of the approach [10] [11] [12], which is named as "Structural theory of the physical world" or simply the Structural theory (ST), a new interpretation of the genesis of $\alpha$ is put forward, a con- 
nection is established between the mass of the EP and $\alpha$, a variant of the classification of EP by masses is proposed.

\section{The Equations of Motion and Mass of Physical Bodies}

In the ST, a hypothesis is accepted on the existence of some particles of the conventionally smallest hierarchical level ( $\varepsilon$-particles), the elementary act of interaction between which (the $\varepsilon$-act) takes place with a strictly defined duration at a strictly defined distance, while due to the $\varepsilon$-act, the particles displace at the same distance. Hence, the number of successively realized $\varepsilon$-acts can be used as a single tool for determining both length and time. Taking the interval of length and time associated with the $\varepsilon$-act ( $\varepsilon$-interval) equal to $\xi_{d} \mathrm{~cm}$ and $\xi_{\tau}$ sec, respectively, let us give a dimensional definition of the smallest path $\xi_{d}$ and time $\xi_{\tau}$ intervals, as a result of this they are called the coefficients of the dimensions of length and time.

$\Delta$-elements of three types are modeled from $\varepsilon$-particles: $\Delta_{i}, \Delta_{j}$, and $\Delta_{k}$ which perform oscillatory motion in mutually perpendicular directions with amplitude $H_{\Delta}$.

A pair of identical $\Delta$-elements (type $2 \Delta_{i}, 2 \Delta_{j}, 2 \Delta_{k}$ ) is characterized by $\alpha_{0}$-multiple repetitions of the amplitude

$$
H_{\Delta}^{2}=H_{c}
$$

that is, the total number of $\varepsilon$-acts

$$
H_{00}=\alpha_{0} H_{c}
$$

where

$$
\alpha_{0}=\sum_{n=1}^{7} n^{2}=\sum_{n=1}^{7} \sum_{l=0}^{n-1}(2 l+1)=140
$$

constant determined by the number of sequentially realized states $\Delta$-pairs, $n$ and $l$ are the analogs of the principal and azimuthal quantum numbers for the considered hierarchical level.

Of the six $\Delta$-elements, the $\gamma$-particles are modeled, which are the basis of the known EPs (electrons, muons, mesons, proton, neutron, etc.), as well as $\gamma_{0 i}$ particles of the general $\Delta$-composition $2 \Delta_{i}, 2 \Delta_{j}, 2 \Delta_{k}$, the presence of which explains the common quantitative laws their movements in all physical bodies.

The resulting trajectory of motion of $\gamma_{0 i}$-particles, determined by excluding time from the periodic parametric equations describing the behavior of each $\Delta$-pair separately, is formed as a torus, the volume of which is called the trajectorial one and is determined by the integrals

$$
\int_{L} S \mathrm{~d} l=\int_{S_{j}} \operatorname{curl} S \mathrm{~d} S_{j}
$$

where $S=S_{i}+S_{k}$, the axial vectors $S_{i}, S_{k}$ and $S_{j}$ are defined using the vector products $H_{j} \times H_{k}, H_{i} \times H_{j}$ and $H_{k} \times H_{i}$, and $\mathrm{d} l=\mathrm{d} l_{i}+\mathrm{dl}_{k}$ is the sum of elementary paths due to $2 i$ - and $2 k$-pairs. 
The above equation differs from the Stokes equation in that it describes the motion of one particle and with its help, the volume is computed: by the circulation of an axial vector $S$ along a closed path (the left side of the equation) or a mixed product of three vectors, one of which is curls (the right side of the equation). The numerical values of the circulation path $L$, axial vectors $S_{i}, S_{k}$ and $S_{j}$, determined using curvilinear integrals [12], are respectively equal to:

$$
L=\alpha_{0} 2 \pi r_{c}, \quad S=\alpha_{0} \pi r_{c}^{2}, \quad S_{j}=\alpha_{0} 4 \pi r_{c}^{2}
$$

where the moduli of oscillations of pairs are taken to be equal to each other: $\left|H_{i}\right|=\left|H_{j}\right|=\left|H_{k}\right|=H_{c}$, besides, it is indicated that

$$
r_{c}=H_{c} \xi_{d} / 2
$$

Let a circle be inscribed in a square with the side $H_{c} \xi_{d}$. Multiplying the length $4 H_{c} \xi_{d}$ and area of this square $H_{c}^{2} \xi_{d}$ by $\pi / 4$, we obtain the circumference inscribed in the square and the area of the circle. Hence, we can conclude that with the help of the coefficient $\pi / 4$ we pass from the spent $\varepsilon$-acts to the measured quantities related to the formation of rounded trajectories of motion. The formulas of the series (5) characterize a planar figure, at the same time, the trajectory of motion of $\gamma_{0 i}$-particles formed by adding three mutually perpendicular oscillations is a three-dimensional figure, that is, a greater number of $\mathcal{\varepsilon}$-acts are spent on the formation of a real trajectory of motion. Taking this circumstance into account, it is assumed that in the transition from three-dimensional to flat figures, the amplitude $H_{c}$ of the oscillation decreases by the factor $\chi_{c}$, thereby introducing a new constant $H_{0}$, we transform Equation (2) to the form

$$
H_{0}=\alpha_{0} H_{c} / \chi_{c}=\alpha_{c} H_{c}
$$

where is denoted

$$
\alpha_{c}=\alpha_{0} / \chi_{c}
$$

Taking into account the designations (5) and (7), we represent the formulas of series (5) in the form

$$
L=\alpha_{c} 2 \pi R, \quad S=\alpha_{c} \pi r_{c}^{2}, \quad S_{j}=\alpha_{c} 4 \pi r_{c}^{2}
$$

The trajectorial volume of $\gamma_{0 i}$-particles obtained from integrals (4) taking into account formulas (9) and interaction conditions are determined by the equations

$$
\begin{gathered}
\alpha_{c} 2 \pi r_{c}\left[\alpha_{c}^{2} \pi r_{c}^{2}\right]=\alpha_{c} \pi \frac{r_{c}}{2}\left[\alpha_{c}^{2} \pi 4 r_{c}^{2}\right]=2 \pi^{2} \alpha_{c}^{3} r_{c}^{3} \\
\alpha_{c} 2 \pi R\left[\pi H_{i}^{2}\right]=\alpha_{c} \pi \frac{r_{c}}{2}\left[\alpha_{c}^{2} \pi 4 r_{c}^{2}\right] \\
\lambda\left[H_{i}^{2}\right]=H_{i}\left[\pi \alpha_{c}^{2} H_{c}^{2}\right]=H_{i}\left[\pi H_{0}^{2}\right] \\
\left(\alpha_{c} 2 \pi R_{c}+\lambda\right)\left[H_{i}^{2}\right]=\left(H_{0}+H_{i}\right)\left[\pi H_{0}^{2}\right]
\end{gathered}
$$

where oriented surfaces (axial vectors) are taken in square brackets.

Equation (10) describes the motion of $\gamma_{0 i}$-particles during intrinsic interac- 
tion involving only $\Delta$-eigenpairs with equal moduli of oscillation amplitudes: $\left|H_{i c}\right|=\left|H_{j c}\right|=\left|H_{k c}\right|=H_{c}$, where the additional subscript " $c$ " marks the characteristics of eigenpairs. With its interaction, the trajectory of motion of $\gamma_{0 i}$ -particles is a torus with equal radii $r_{c}=H_{c} \xi_{d} / 2$. With the aid of Equation (11), the motion of $\gamma_{0 i}$-particles involved in the interaction with their own partner is described. As an example of this type of interaction, one can consider the electric and gravitational interactions [10] [11]. In this case, the interaction mechanism is reduced to the following: the proper particles are making the shuttle motion relative to their bases and form complexes with the bases of their partners, exchanging $\Delta$-pairs. Particles with the participation of which the electrostatic interaction is realized at a distance are called $\gamma_{E}$-particles, the index " $E$ " at $\gamma$ indicates the nature of the interaction. With the help of the index " $i$ " at the amplitude $H_{i}$ of the oscillation in Equations (10)-(13), it is indicated the participation in the interactions of its own or third-party partners. When $H_{i}<H_{c}$ the trajectory of motion is in the form of a torus with the large radius $R$ and the small radius $r=H_{i} \xi_{d} / 2$.

Equation (11) is often convenient to represent in the form

$$
\alpha_{c} 2 \pi R\left[H_{i}^{2}\right]=\alpha_{c} H_{c}\left[\alpha_{c}^{2} \pi H_{c}^{2}\right]
$$

Interactions with third-party partners are implemented mainly by external influences: mechanical, radiation, heat transfer, etc. The results of interaction are reduced to the transfer or exchange of doublets from $\gamma_{0 i}$-particles ( $\beta_{\varepsilon}$-pairs) of third-party origin. In a free state, $\beta_{\varepsilon}$-pairs are photons characterized by the $\Delta$ compositions of the type $2 j 2 \bar{i} 2 k / 2 \bar{j} 2 i 2 k$ [10] [11], where the dashes above the $\Delta$-element indicate that the $\Delta$-pair is moving in the opposite direction.

The presence in the numerator and denominator of $\Delta$-pairs with the same directions and phases of motion determines the direction of the photon motion, while $\lambda$ in Equation (12) it is not the wavelength at all, this is the smallest transverse path, with the passage of which the manifestation of $\beta_{\varepsilon}$-pairs (or photon) as an integral formation. In various media $\gamma_{0 i}$-particles from outside $\beta_{\varepsilon}$-pairs form complexes with the particles of the medium, the motion of which is already described by the Equation (13).

In [10] [11] it was shown that proceeding from the equations of motion (4), (10-14) it is possible to derive all the equations of classical mechanics, electrodynamics, and thermodynamics having an empirical origin.

From the quantitative laws of motion (10-14) it follows that the smallest intervals of the longitudinal and transverse paths associated with the manifestation of the integrity of the $\gamma_{0 i}$-particles are equal to

$$
\begin{gathered}
l_{i}=H_{i} \xi_{d}, l_{0}=H_{0} \xi_{d}, l_{0 i}=\left(H_{0}+H_{i}\right) \xi_{d} \\
\lambda=\frac{\pi H_{0}^{2} \xi_{d}}{H_{i}}, \lambda_{0}=\frac{\pi H_{0}^{2} \xi_{d}}{H_{o}}=\pi H_{0} \xi_{d}, \lambda_{0 i}=\frac{\pi\left(H_{0}+H_{i}\right) H_{0}^{2}}{H_{i}^{2}}
\end{gathered}
$$

The corresponding time intervals for longitudinal and transverse motion are the same and determined by the formulas 


$$
\tau_{i}=H_{i} \xi_{\tau}, \tau_{0}=H_{0} \xi_{\tau}, \tau_{0 i}=\left(H_{0}+H_{i}\right) \xi_{\tau}
$$

where taking into account the fact that during longitudinal displacement a certain number of $\mathcal{\varepsilon}$-intervals is spent on weaving a perpendicular surface, a new time coefficient is introduced

$$
\xi_{\tau}=\pi H_{0}^{2} \xi_{t}
$$

Based on Equations (15) and (16), the speed of longitudinal motion is determined by the formula

$$
v=\frac{H_{i}}{H_{0}+H_{i}} c
$$

where is denoted

$$
c=\frac{\xi_{d}}{\xi_{\tau}}
$$

Using formulas (16), (19), and (20), we compose the following identities $\lambda c \equiv \lambda c, \lambda_{0} c \equiv \lambda_{0} c, \lambda v \equiv \lambda v$ and represent their right-hand sides as a product of dimensional and dimensionless components

$$
\begin{aligned}
& \lambda c=\frac{\pi H_{0}^{2} \xi_{d}^{2}}{H_{i} \xi_{\tau}}, \lambda_{0} c=\pi H_{0} \frac{\xi_{d}^{2}}{\xi_{\tau}}, \\
& \lambda v=\frac{\pi H_{0}^{2} H_{i}}{H_{i}\left(H_{0}+H_{i}\right)} \frac{\xi_{d}^{2}}{\xi_{\tau}}=\frac{\pi H_{0}^{2}}{\left(H_{0}+H_{i}\right)} \frac{\xi_{d}^{2}}{\xi_{\tau}}
\end{aligned}
$$

Dividing and multiplying the right-hand sides of the given equalities by a strictly constant value with the dimension of mass $\xi_{m}$, we obtain:

$$
\lambda c=h m_{i}^{-1}, \lambda_{0} c=h m_{0}^{-1}, \lambda v=h m^{-1}
$$

or

$$
m_{i} c \lambda=h, m_{0} c \lambda=h, m v \lambda=h
$$

where the following is denoted:

$$
\begin{gathered}
m_{i}=\frac{\xi_{m} H_{i}}{\pi H_{0}^{2}}, m_{0}=\frac{\xi_{m} H_{0}}{\pi H_{0}^{2}}, m=\frac{\xi_{m}\left(H_{0}+H_{i}\right)}{\pi H_{0}^{2}} \\
h=\frac{\xi_{m} \xi_{d}^{2}}{\xi_{\tau}}
\end{gathered}
$$

From formula (25) it follows that the constant $h$ is a combination of the dimension coefficients; it is shown in the ST that $h$ is numerically equal to Planck's constant.

The symbol $m_{i}$ denotes the mass of interaction with third-party partners, the mass of "rest" $m_{0}$ is the result of its interaction, $m$ is the total mass, which can be represented as the sum

$$
m=m_{0}+m_{i}
$$

By analogy with $\xi_{d}$ and $\xi_{\tau}$, the constant $\xi_{m}$ is called the coefficient of a dimension of the mass. Many other dimensions can be combined with $\xi_{d}$, $\xi_{\tau}$ 
and $\xi_{m}$.

Integrating from $\xi_{d}, \xi_{\tau}$ and $\xi_{m}$ the energy dimension coefficient

$$
\xi_{\varepsilon}=\frac{\xi_{m} \xi_{s}^{2}}{\xi_{\tau}}
$$

and multiplying by dimensionless components of a certain mass (24), and taking into account (26), we obtain

$$
m_{i} c^{2}=\frac{\xi_{\varepsilon} H_{i}}{\pi H_{0}^{2}}, m_{0} c^{2}=\frac{\xi_{\varepsilon i}}{\pi H_{0}}, m c^{2}=\frac{\xi_{\varepsilon}\left(H_{0}+H_{i}\right)}{\pi H_{0}^{2}},
$$

whence it follows that the content of the unity of mass and energy is in the unity of their dimensionless components.

By slightly transforming Equations (16), (22), and (28), the energy of $\gamma_{0 i}$ -particles can be represented as the reciprocal of the transverse path:

$$
m_{i} c^{2}=\frac{c h}{\lambda}, m_{0} c^{2}=\frac{c h}{\alpha_{c} 2 \pi r_{c}}, m v^{2}=\frac{c h}{\alpha_{c} 2 \pi r+\lambda},
$$

where taking into account formulas (20), (25), and (27), we denote

$$
\xi_{\varepsilon} \xi_{d}=c h
$$

Based on Equations (21), (24), (28), and (29), we can conclude that the fundamental property of matter, mass, is determined by the ratio of the perpendicular surfaces of transverse motion to the value of the corresponding trajectory volumes-the reciprocal of the transverse path.

We emphasize that the above definitions refer to inertial mass.

\section{Numerical Values of Dimension Coefficients, $\alpha_{c}$ and $H_{c}$}

It was shown in [12] and [13] that the Newton gravity constant $G$ is also a combination of the dimension coefficients:

$$
G=\frac{\xi_{d}^{3}}{2 \pi \xi_{m} \xi_{\tau}^{2}},
$$

whence, taking into account formulas (20) and (25), we obtain the connection between $\xi_{d}, \xi_{\tau}, \xi_{m}$ and world constants $c, h, \hbar=h / 2 \pi$ and $G$ :

$$
\begin{aligned}
& \xi_{d}=\left(\frac{2 \pi G h}{c^{3}}\right)^{1 / 2}=1.015 \times 10^{-34} \mathrm{~m}, \\
& \xi_{\tau}\left(\frac{2 \pi G h}{c^{5}}\right)^{1 / 2}=3.38 \times 10^{-43} \mathrm{~s} \\
& \xi_{m}=\left(\frac{c h}{G}\right)^{1 / 2}=2.176 \times 10^{-8} \mathrm{~kg}
\end{aligned}
$$

Because an electron in the state of proper interaction consists of two $\gamma_{0 i}$ -particles [10] [11], taking into account the second equality (24), its mass $m_{e}$ is determined by the formula

$$
m_{e}=\frac{2 \xi_{m}}{\pi H_{0}}=\frac{\xi_{m}}{H_{e 0}},
$$


respectively, we represent the second formula of series (29) in the form

$$
m_{e} c^{2}=\frac{e h}{\alpha_{c} 2 \pi r_{e}}
$$

where the following notation is used

$$
H_{e 0}=H_{0} / 2, r_{e}=r_{c} / 2
$$

Using formulas (32) and (33), we determine the numerical values of the constants $H_{e 0}$ and $H_{0}$ :

$$
H_{e 0}=7.6034 \times 10^{21}, H_{0}=1.5207 \times 10^{22}
$$

where $m_{e}$ is taken as $m_{e}=9.11 \times 10^{-31} \mathrm{~kg}$ [14].

Multiplying and dividing the right side of Equation (34) by the square of a constant value with the dimension of the charge $\xi_{q}$, that is, by the factor of the dimension of the charge, we obtain

$$
m_{e} c^{2}=\frac{c \hbar \xi_{q}^{2}}{\alpha_{c} \xi_{q}^{2} r_{e}}=\frac{\xi_{q}^{2}}{r_{e}},
$$

where the numerical value $\xi_{q}^{2}$ is selected in such a way that the following condition is met

$$
\frac{c \hbar}{\alpha_{c} \xi_{q}^{2}}=1
$$

Comparing formula (37) with the well-known formula for the proper interaction of an electron $m_{e} c^{2}=e^{2} / r_{e}$, we can conclude that the coefficient of dimensionality of the charge $\xi_{q}$ is equal to the value of the electric charge, that is,

$$
\xi_{q}=e
$$

Hence, taking into account condition (38), it follows that

$$
\alpha_{c} \xi_{q}^{2}=c \hbar=\alpha_{c} e^{2}
$$

or

$$
\alpha_{c}=\alpha^{-1}
$$

that is, the constant $\alpha_{c}$ determined using formulas (3) and (8) is numerically equal to the reciprocal of the fine structure constant $\alpha$.

Based on (7), (36), and (41) we determine the numerical value of the constant $H_{c}=1.11 \times 10^{20}$, whence, taking into account formulas (6), (32), and (35), we obtain the value of the classical radius of the electron,

$$
r_{e}=\frac{H_{c} \xi_{d}}{4}=2.82 \times 10^{-15} \mathrm{~m}
$$

which is an additional confirmation of the results (39), and (41).

Transforming the equation of motion (14) to the form

$$
\frac{H_{i}^{2}}{H_{o} \pi H_{0}^{2}}=\frac{\xi_{d}}{\alpha_{c} 2 \pi r}
$$

taking into account (19), (28), (39) and (40), we obtain the equation for the elec- 
trical interaction of unit charges

$$
\varepsilon_{q}=m v^{2}=\frac{\xi_{\varepsilon} \xi_{d}}{\alpha_{c} 2 \pi r}=\frac{c h}{\alpha_{c} 2 \pi r}=\frac{e^{2}}{r},
$$

whence formula (40) and equality (41) follow again.

In [11] it is shown that the dimensional value of the charge $q$ is determined by the product of the number $N_{q}$ of $\gamma_{E}$-particles and the coefficient of dimension $\xi_{q}$, that is, $q=N_{q} \cdot \xi_{q}$; thus, taking into account formula (44), the Coulomb equation for charges $q_{1}$ and $q_{2}$ is represented by the formula

$$
\varepsilon_{q}=\frac{N_{q 1} N_{q 2} \xi_{q}^{2} \xi_{d}}{r}=\frac{N_{q 1} N_{q 2} e^{2}}{r}=\frac{q_{1} q_{2}}{r}
$$

Four important conclusions follow from the derivation of the Coulomb equation:

- the magnitude of the elementary charge $e$ is equal to the dimension coefficient of the charge $\xi_{q}$

- the square of the elementary charge $e^{2}$, introduced based on the Coulomb equation to give the property of matter the "charge" of dimensional content is the essence of a combination of constants $\alpha_{c}, 2 \pi$ and coefficients of dimensions $\xi_{s}, \xi_{\tau}$ and $\xi_{m}$ :

$$
e^{2}=\frac{\xi_{m} \xi_{d}^{3}}{\alpha_{c} 2 \pi \xi_{\tau}^{2}}
$$

- the constant $\alpha_{c}^{-1}$ is numerically equal to the fine structure constant $\alpha_{c}=\alpha^{-1}$

- the radius of the closed transverse path is the distance of interaction of the number charge and structure formation.

From series (23) in integral form follows the basic equation of mechanics

$$
m v=m_{i} c,
$$

according to which the momentum $P$ is uniquely determined by the interaction mass in the corresponding direction. Both momentum $P$ and paths $l_{i}(15)$ and $\lambda$ (16) are criteria for identifying the particle under consideration and are related to the realization of the same variable:

$$
l_{i}=H_{i} \xi_{d}, \lambda=\pi H_{0}^{2} \xi_{d} / H_{i}, P=\xi_{p} H_{i} / \pi H_{0}^{2},
$$

where $\xi_{p}=\xi_{m} c$ is the coefficient of a dimension of momentum. From (48) it follows that $\lambda$ is inversely proportional to $H_{i}$, while the momentum is directly proportional; thus the fulfillment of the condition $m v \lambda=m_{i} c \lambda=h$ becomes a condition for the manifestation of the integrity of the particle. Accordingly, when passing a transverse path $n \lambda$, where $n=1,2,3, \cdots$, the integer number of particles appears $n$ time, that is $m v n \lambda=n h$. If the path is equal to $n \lambda$ : to the value of a closed transverse path, for example, a circle with a length, then

$$
2 \pi r=n \lambda \text { or } m v r=n \hbar
$$

Whence, taking into account Equations (11) and (7), it follows

$$
n \cdot H_{i}=H_{c} \text { или } n \alpha_{c} H_{i}=H_{0}
$$


Replacing the variable $H_{i}$ by a variable $n$ using formula (50), we represent Equations (11), (12), (49), and (35) in the form of the Bohr relations characterizing the stationary orbits of an electron in a hydrogen atom.

$$
\begin{gathered}
\lambda=2 \pi n \alpha_{c}^{2} r_{e}, 2 \pi r=2 \pi n^{2} \alpha_{c}{ }^{2} r_{e} \\
v=\frac{c}{n \alpha_{c}+1} \approx \frac{c}{n \alpha_{c}}, m_{e} v r=n \hbar, \varepsilon=\frac{m_{e} c^{2}}{2 n \alpha_{c}\left(n \alpha_{c}+1\right)} \approx \frac{m_{e} c^{2}}{2 n^{2} \alpha_{c}^{2}}
\end{gathered}
$$

From the above results, it follows that the orbits of the electron are trajectories of transverse motion, while the principal quantum number indicates the multiplicity of manifestation of the integrity of the electron in stationary orbits. Taking into account the first formula (51), the quantity $\lambda$ can be represented as the sum of transverse paths $\lambda_{i}$ and $\lambda_{k i}$ caused by the proper $2 i$ - and $2 k$-pairs.

$$
\lambda=\lambda_{i}+\lambda_{k i}=\pi\left(n_{i}+n_{k i}\right) \alpha_{c}^{2} H_{e c} \xi_{d}=\pi n \alpha_{c}^{2} H_{e c} \xi_{d}
$$

where is denoted

$$
n_{i}+n_{k i}=n
$$

$n_{i}$ and $n_{k i}$ indicate the multiplicity of manifestation of the corresponding transverse paths and are called the Eigenquantum numbers. From Equation (54) and the equality of the moduli of the amplitudes $\left|H_{i c}\right|=\left|H_{k c}\right|$ follows the always fulfilled condition

$$
n_{i}=n_{k i}
$$

At $n_{i}+n_{k i}=1$, condition (55) implies

$$
n_{i}=n_{k i}=0.5
$$

In the case of excited $\mathrm{H}$ atoms, the $\gamma_{0 i}$-particles of photons absorbed by the atom form complexes with the electron under consideration, followed by exchange $2 k$ - or $2 j$-pairs, and under new $\Delta$-compositions, new trajectories of motion are formed. By analogy with its interaction, introducing the transverse paths $\lambda_{\mathrm{kf}}=\pi n_{\mathrm{kf}} \alpha_{c}^{2} H_{c e} \xi_{d}$ and $\lambda_{j}=\pi n_{j} \alpha_{c}^{2} H_{c e} \xi_{d}$ caused by $2 k_{f}$ and $2 j$-pairs, the final electron orbit is represented by the equation

$$
n\left(\lambda_{i}+\lambda_{k i}+\lambda_{k f}+\lambda_{j}\right)=n \pi\left(n_{i}+n_{k i}+n_{k f}+n_{j}\right) \alpha_{c}^{2} H_{e c} \xi_{d}=\pi n^{2} \alpha_{c}^{2} H_{e c} \xi_{d},
$$

where proper and third-party $\Delta_{k}$-pairs are marked with additional indices " 1 " and " $f$ ", respectively, and the following is denoted

$$
n=n_{i}+n_{k i}+n_{k f}+n_{j}
$$

Equation (57) implies that for $n_{k f}=n_{j}=0$, trajectories are formed in the form of circles, at $n_{j}=0$, we have elliptical orbits, the condition $n_{j} \neq 0$ indicates the orientation of the orbits relative to the plane formed by $2 i$ - and $2 k$-pairs.

From equation (58) and condition (56), it follows that for a given $n$, the sum $n_{k f}+n_{j}$, like the azimuthal quantum number 1 , takes $n-1$ values, that is,

$$
m_{l}=n_{k f}+n_{j}
$$


From this, it follows that the content that $l$ is related to interactions with the participation of third-party partners, while $n_{k f}$, as well as $n_{j}$, can take from zero to $l$-values, that is, the sum $n_{k f}+n_{j}$ can be realized by $2 l+1$ variants. It is easy to verify that we obtain the indicated number of variants by determining the magnetic quantum number by the difference

$$
m_{e}=n_{k f}-n_{j}
$$

that is, given $I$ the participation of third-party partners, it is possible to form a subgroup with $2 l+1$ different states.

Just as $n_{i}$ and $n_{k i}$ are called eigen quantum numbers, $n_{k f}$ and $n_{j}$ are called the third-party quantum numbers, because their origin is related to the participation of third-party partners in interactions.

Taking into account condition (55), the proposed set of quantum numbers $n_{i}, n_{k i}, n_{k f}$ and $n_{j}$ are not only an alternative to quantum numbers $n, l$ and $m_{l}$, but also reveals an additional aspect of their content-the $\Delta$-content is determined, therefore, the trajectory of motion of the composite $\gamma_{0 i}$-particlesthe nature and number of interacting partners is indicated because each $\Delta$-content is formed with the participation of a specific partner by interaction.

The total number of possible options for interaction, respectively, and the possible number of partners is determined by the sum $\sum_{n} \sum_{l=0}^{n-1}(2 l+1)$. For example, this sum characterizes the state of an electron in many-electron atoms and nucleons in nuclei (shell theory of the nucleus [15]). In [10], it is shown that systems of eight units (initially given by seven partners) predetermine the patterns of formation of complete families, ranging from $\Delta$-elements from $\varepsilon$-particles to subsequent generations of a higher hierarchical level, including for many-electron atoms and nuclei. The sum (3) becomes a completeness criterion for a particular hierarchical generation in terms of the number of possible formations.

\section{The Classification of Elementary Particles by Masses}

From the content of the constant $\alpha_{0}$ it follows that with the participation of the correction (8), it can be used as a basis for determining the mass of the elementary particles and their classification by masses. To this end, introducing the interaction

$$
H_{i}=n^{p} \alpha_{c}^{q} H_{0},
$$

it is possible to compose a set of energy scales with a characteristic classification parameter $q=0, \pm 1, \pm 2$, each of which, in turn, using the principal and azimuthal quantum numbers, is divided into groups and corresponding subgroups. The parameter $p$ in (61) indicates an increase $(p=+1)$ or a decrease $(p=-1)$ in the interaction potential relative to the initial potential of a particular energy scale. Thus, in addition to the generally accepted set of quantum numbers, the number $q$ is additionally introduced, which becomes a characteristic parameter 
for the classification of composite elements, elementary particles, over the energy.

Introducing (61) into Equation (14), we obtain the dependence of the interaction distance on the parameter $q, n$ and $p$ :

$$
R=\frac{H_{e} \xi_{d}}{2 n^{2 p} \alpha_{c}^{2 q}}
$$

where the numerical value $\xi_{d}$ is given in the series (32).

Thus, the intrinsic spectrum of interaction distances corresponds to a specific energy scale. The energy scales with $q=-2$ and $q=-1$ (the interaction distance $1.25 \times 10^{-5} \div 6.65 \times 10^{-10} \mathrm{~m}$ ) are characterized by electromagnetic interactions in atomic-molecular systems and systems of the nucleus-electron type. In particular, for the energy scale $q=-1$ at $p=-1$, taking into account formula (50), we obtain the Bohr Equations (51) and (52) characterizing the stationary orbits of the hydrogen atom.

It should be noted that elementary particles are not directly formed as a result of electrostatic and magnetic interactions; only under certain conditions, after certain transformations, the photons without the rest mass are born

Based on definitions (24), (26), and (61), the mass of one $\gamma_{0 i}$-particle is given by the equation

$$
m=\left(1+n^{p} \alpha_{c}^{q}\right) m_{0}
$$

at small interaction distances, when $q \geq 1$ and $n^{p} \alpha_{c}^{q} \gg 1$, the mass is determined mainly by the third-party interactions.

$$
m=n^{p} \alpha_{c}^{q} m_{0}
$$

Accordingly, the masses of elementary particles, taking into account the number of -particles $N_{\gamma}$ in their composition, are described by the equations

$$
m=\left(1+N_{\gamma} n^{p} \alpha_{c}^{q}\right) m_{0} \text { or } m=N_{\gamma} n^{p} \alpha_{c}^{q} m_{0}
$$

As already noted, in the framework of energy scales with negative $q$ values, the photons without a "rest" mass are mainly described. Now let us present a Table,

\begin{tabular}{|c|c|c|c|c|c|c|c|c|}
\hline \multirow{2}{*}{$q$} & \multirow{2}{*}{$p$} & \multicolumn{7}{|c|}{$n$} \\
\hline & & 1 & 2 & 3 & 4 & 5 & 6 & 7 \\
\hline \multirow{3}{*}{0} & +1 & \multirow{3}{*}{0.2555} & 0.511 & 0.7665 & 1.022 & 1.2775 & 1.533 & 1.7885 \\
\hline & & & & & & & & \\
\hline & -1 & & 0.12775 & 0.08516 & 0.064 & 0.0511 & 0.04258 & 0.0365 \\
\hline \multirow{3}{*}{1} & +1 & \multirow{3}{*}{35.0127} & 70.0254 & 105.0381 & 140.051 & 175.064 & 210.076 & 245.0888 \\
\hline & & & & & & & & \\
\hline & -1 & & 17.506 & 11.671 & 8.753 & 7.003 & 5.835 & 5.008 \\
\hline \multirow{3}{*}{2} & +1 & \multirow{3}{*}{4798} & 9596 & 14,396 & 19,192 & 23,990 & 28,788 & 33,586 \\
\hline & & & & & & & & \\
\hline & -1 & & 2399 & 1599 & 11,995 & 959.6 & 799.6 & 685 \\
\hline
\end{tabular}
where the masses of the individual $\gamma_{0 i}$-particles are given in $\mathrm{MeV}$ units, starting with $q=0$ (Table 1 ).

Table 1 . The values $q, n$ and the mass of the $\gamma_{0 i}$-particles. 
$q=0$. From the $\gamma_{0 i}$ particles of this scale, one can model the lightest lepton (electron) with mass $m_{e}=2 m_{0}\left(N_{\gamma}=2, n=1\right)$, estimate the masses of the first generation quarks $\mathrm{u}$ and $\mathrm{d}: \approx 2 \mathrm{MeV}$ and $\approx 4 \mathrm{MeV}$, accordingly, to operate with the difference between the masses of the neutron $\left(\mathrm{n}^{0}\right)$ and proton $\left(\mathrm{p}^{+}\right)-1.23$ $\mathrm{MeV}$, estimate the mass defect (i.e., the binding energy) between nucleons, starting from the deuterium nucleus $(2.23 \mathrm{MeV})$ and up to the heavy nuclei $(\approx 7$ $9 \mathrm{MeV}$ per nucleon)

$q=1 . \gamma_{0 i}$-particles of this scale are most promising for determining the mass of muonic leptons $(104-106 \mathrm{MeV})$ and $\tau$-lepton $(1777 \mathrm{MeV})$, strange s-quark $(104 \mathrm{MeV})$ and charmed c-quark $(1270 \mathrm{MeV}), \pi$-mesons $(137-140 \mathrm{MeV}), \mathrm{p}^{+}$ (938.28) and $n^{0}(939.57 \mathrm{MeV})$, the constant of the energy scale of quantum chromodynamics $\Lambda_{Q C D}\left(217_{-23}^{+25} \mathrm{MeV}\right)$.

$q=2$. Particles of this energy scale can be used to simulate the b quark (4.2 $\mathrm{GeV}$ ) and the top t quark $(173 \mathrm{GeV})$, but basically, this group covers the energy parameters of the weak interaction [16], the Higgs scalar field constant $(<\mathrm{H}\rangle=247$ $\mathrm{GeV})$, the masses of Higgs bosons $(24.3-125 \mathrm{GeV})$, gauge bosons $\mathrm{W}^{ \pm}(80 \mathrm{GeV})$, vector boson $\mathrm{Z}(91 \mathrm{GeV})$, heavy bosons $\mathrm{W}_{\mathrm{r}}$ and $\mathrm{Z}_{\mathrm{CM}}(405$ and $505 \mathrm{GeV})$ [17], etc. In the Standard Model of elementary particle physics (SM), it is just with processes in the energy region at $q=2$ according to the Higgs scheme that the mechanism of production of massive elementary particles: $\mathrm{W}^{ \pm}$and Z-bosons is explained.

It follows from Equation (29) that it is impossible to obtain particles with the mass of $\xi_{m} \approx 1.22 \times 10^{19} \mathrm{GeV}$ or $\xi_{m} / \alpha \approx 6.5 \times 10^{14} \mathrm{GeV}$ (respectively, the energy scales of Superstring theory [18] and the Grand Unification [19] because for this purpose it is necessary to approach an unrealizable condition $H_{i S}^{2}=H_{0}^{3}$. The values of the given order of magnitudes can only be the auxiliary parameters when carrying out the computing operations.

In general, by appropriate selection $N_{\gamma}, q$ and $p$, it is possible to compute the mass of almost any elementary particle. However, to avoid arbitrariness and errors, first of all, it is necessary to take into account the $\gamma_{0 i}=$ composition of the considered elementary particles, information about which can be obtained from the experimentally observed results of theirs decay. Besides, based on the proposed model, it will be very useful to determine besides the mass the other characteristics of given elementary particles: spin, magnetic moment, etc. It is not excluded that particles with very close masses can be formed by different combinations of $N_{\gamma}, q$ and $p$, respectively, and the properties of these particles, especially their decay products, will be very different. As an example, consider the production of $\pi^{ \pm}$-mesons from accelerated electron-positron pairs according to the scheme

$$
e^{-}+e^{+} \rightarrow \pi^{-}+\pi^{+},
$$

and their subsequent decay through the channels

$$
\begin{aligned}
\pi^{ \pm} & \rightarrow \mu^{+}+v_{\mu} ; \mu^{+} \rightarrow e^{+}+v_{e}+\tilde{v}_{\mu} \\
\pi^{-} & \rightarrow \mu^{-}+\tilde{v}_{\mu} ; \mu^{-} \rightarrow e^{-}+\tilde{v}_{e}+v_{\mu}
\end{aligned}
$$


From the particle balance it follows that as a result of the above transformations, six additional neutrino particles were formed: electron neutrinos $v_{e}$ and antineutrinos $\tilde{v}_{e}$, and two muonic neutrinos $v_{\mu}$ and antineutrinos $\tilde{v}_{\mu}$. When explaining the indicated and any other transformations with the participation of elementary particles in ST, one does not operate with the ideas of physical vacuum and secondary quantization. By analogy with chemistry, any transformations with the participation of elementary particles are considered as processes of connection, decay, and exchange of constituent elements. The very process of acceleration of electrons and positrons is reduced to the formation of complexes with the participation of particles of the accelerating medium and accelerated charges, thus the already mentioned complexes participate in collisions. In particular, decays of $\pi^{+}$and $\pi^{-}$mesons through channels (67) and (68) are represented by the following schemes:

$$
\begin{gathered}
e^{+} \beta_{\varepsilon 1} \beta_{\varepsilon 2} \gamma_{e v} \rightarrow e^{+} \beta_{\varepsilon 1} \tilde{\gamma}_{\mu v} \gamma_{e v}+v_{\mu} \\
e^{+} \beta_{\varepsilon 1} \tilde{\gamma}_{\mu \gamma} \gamma_{e \gamma} \rightarrow e^{+} \beta_{\varepsilon 1}+\tilde{v}_{\mu}+v_{e} \\
e^{-} \beta_{\varepsilon 1} \beta_{\varepsilon 2} \tilde{\gamma}_{e v} \rightarrow e^{-} \beta_{\varepsilon 1} \gamma_{\mu \nu} \tilde{\gamma}_{e v}+\tilde{v}_{\mu} \\
e^{-} \beta_{\varepsilon 1} \beta_{\varepsilon 2} \tilde{\gamma}_{e v} \rightarrow e^{-} \beta_{\varepsilon 1} \gamma_{\mu v} \tilde{\gamma}_{e V}+\tilde{v}_{\mu}
\end{gathered}
$$

where $e^{+} \beta_{\varepsilon 1} \beta_{\varepsilon 2} \gamma_{e v}-\pi^{+}$-muons, $e^{-} \beta_{\varepsilon 1} \beta_{\varepsilon 2} \tilde{\gamma}_{e v}-\pi^{-}=$meson, $e^{+} \beta_{\varepsilon 1} \tilde{\gamma}_{\mu v} \gamma_{e v}$ and $e^{-} \beta_{\varepsilon 1} \gamma_{\mu \nu} \tilde{\gamma}_{e v}$ respectively $\mu^{+}$and $\mu^{-}$muons, $\beta_{\varepsilon 1}$ and $\beta_{\varepsilon 2}$ doublets of $\gamma_{0 i}$ -particles with different potentials $\gamma_{e v}, \tilde{\gamma}_{e v}, \gamma_{\mu v}$ and $\tilde{\gamma}_{\mu v}$ inclusions of six $\Delta$-elements, transforming during decay into an electron and muon neutrinos and antineutrinos, respectively.

As a rule, the potential of $\beta_{\varepsilon 1}$-pairs determines the kinetic energy of the resulting electrons and positrons. The neutrino particles themselves are characterized by $\Delta$-compositions of the type $[2 j(i k)]_{I}(\bar{i} \bar{k})_{I I}$ where the indices "I" and "II" indicate the temporal state of the compound $\Delta$-pairs [10] [11].

According to schemes (69) and (71), $\pi^{+}$and $\pi^{-}$mesons consist of six $\gamma_{0 i}$ -particles, and their mass is represented by the sum

$$
m_{\pi} \approx(4 \times 17.5+2 \times 35) \mathrm{MeV}=140 \mathrm{MeV}
$$

that is from four $\gamma_{0 i}$-particles with the mass $17.5 \mathrm{MeV}\left(N_{\gamma}=4, n=2, p=\right.$ $-1, q=1)$ and two $\mu^{+}$-particles with a mass of $35.5 \mathrm{MeV}\left(N_{\gamma}=2, n=1, p=\right.$ $-1, q=1)$. According to schemes (70) and (72), $\mu^{+}$- and $\mu^{-}$-mesons consist of five $\gamma_{0 i}$-particles with a total mass

$$
m_{\mu^{ \pm}} \approx(4 \times 17.5+35.5) \mathrm{MeV}=105.5 \mathrm{MeV}
$$

That is, the mass of $\mu$-mesons is composed of four $\gamma_{0 i}$-particles with mass 17.5 $\mathrm{MeV}\left(N_{\gamma}=4, n=2, p=-1, q=1\right)$ and from one particle of mass $35.5 \mathrm{MeV}$ $\left(N_{\gamma}=1, n=2, p=-1, q=1\right)$

In [20], the following formula was proposed

$$
m_{\mu}=\frac{3 m_{e}}{2 \alpha}
$$


where the relationship between the masses of electron $\left(m_{e}\right)$, muon $m_{\mu}$, and $\alpha$ is written. Proceeding from the fact that an electron in the state of its interaction consists of two $\gamma_{0 i}$-particles, the given ratio could be explained by the sum of the masses of six $\gamma_{0 i}$-particles with masses of $17.5 \mathrm{MeV}$. Nevertheless, based on the $\mu^{ \pm}$-meson decay schemes (70) and (72), the most probable variant of the definition $m_{\mu}$ is the sum (74).

Based on the composition of the mesons decay products $\pi^{0}, \pi^{0} \rightarrow 2 f_{\gamma}$ where $f_{\gamma}$ are gamma quanta, we can conclude that $\pi^{0}$-meson consists of four $\gamma_{0 i}$-particles with a mass of $35.5 \mathrm{MeV}$ each, that is, the composition of $\pi^{0}$ a meson is very different from $\pi^{ \pm}$mesons.

We do not say at all that with the help of the given Table it is possible to unambiguously determine the mass of any elementary particle, however, concerning a specific elementary particle, the selection of combinations from various options can become an additional factor in compiling its correct model. So, it is very tempting to represent the proton mass $m_{p}$ as the sum

$$
m_{p} \approx 4(210+17.5+7) \mathrm{MeV}=938 \mathrm{MeV}
$$

where groups with $N_{\gamma}=4, q=1$, and $n=6 . p=+1 ; n=2, p=-1 ; n=5, p=-1$ are taken into account.

An interesting option is also

$$
m_{p} \approx 4(217+17.5) \mathrm{MeV} \approx 938 \mathrm{MeV}
$$

where $217 \mathrm{MeV}$ is the energy scale of quantum chromodynamics.

Due to the stability of the proton, taking into account the third formula of a series (24), its mass can be uniquely determined by the relation

$$
m_{p}=\frac{\xi_{m}\left(H_{i}+H_{p}\right)}{\pi H_{0}^{2}}
$$

where $H_{p}$ is the total potential of interactions involving composite $\gamma_{0 i}$ particles of a proton.

Thus, the calculation of the proton mass is reduced to the calculation of the constant $H_{p}$.

We especially note that the mass of $17.5 \mathrm{MeV}$, which appears in formulas (73), (74), (76), and (77), is quite close to the key quantity considered in [21] [22].

From the foregoing, it follows that the proposed method for determining the mass based on the mechanism of motion of physical bodies seems to be a promising basis for computing the masses of elementary particles, regardless of the interactions in which they participate, whether they belong to fermions or bosons, leptons or baryons.

\section{The Characteristic Constants of Interactions and Equations of Motion}

Any motions, the parameters of which determine the mass of physical bodies, are caused by interactions. So, for the electromagnetic interaction, one uses $\alpha$ and $e^{2}$, related by an equation $\alpha=e^{2} / c \hbar$; practically similar formulas are used 
to describe the strong and weak interaction. The gravitational coupling constant $\alpha_{G}$, widely discussed [23], is represented by the formula

$$
\alpha_{G}=\frac{G m^{2}}{c \hbar}
$$

where $m$ is the mass of gravitationally interacting particles.

In particular, $\alpha_{G}$ for the electron $\left(\alpha_{G e}\right)$ and proton $\left(\alpha_{G p}\right)$ are determined by the formulas

$$
\alpha_{G e}=\frac{G m_{e}^{2}}{c \hbar} \text { and } \alpha_{G p}=\frac{G m_{p}^{2}}{c \hbar}
$$

Whence, taking into account formulas (20), (24), (25), (31), and (33) it follows

$$
\alpha_{G e}=\frac{4}{\pi^{2} H_{0}^{2}}, \alpha_{G p}=\frac{H_{p}^{2}}{\pi^{2} H_{0}^{4}}
$$

where $H_{0}=\alpha_{c} H_{c}$ are products of constants $\alpha_{c}$ and $H_{c}$ (7), $H_{i}=H_{p} \gg H_{0}$ is taken into account, $H_{p}$ is the proton interaction potential. The presence of relation between $H_{c}$ and $\alpha_{c}$ directly follows from the basic equations of motion (10)-(14). Namely, by $\alpha_{c}$-multiple changes in the constant $H_{c}$, the energy scales are selected and they can be computed using $\alpha_{c}$ and $H_{c}$.

Similarly, based on formulas (20), (25), and (40), it can be shown that the square of the charge is the product of dimensional and dimensionless constants:

$$
e^{2}=\frac{c \hbar}{\alpha_{c}}=\frac{\xi_{m} \xi_{d}^{3}}{2 \pi \alpha_{c} \xi_{\tau}^{2}}
$$

That is, when choosing the dimension of the property of matter (based on the Coulomb equation), constants $\alpha_{c}=\alpha_{0} / \chi_{c}$ (8) and $2 \pi$ were included in the value of $e^{2}$.

Thus, when describing various interactions and related phenomena, we operate with primary constants of three types: structural content $H_{c}$ and $\alpha_{0}$; geometric origin $\pi$ and $\chi_{c}$; the coefficients of dimensions of length $\xi_{d}$, time $\xi_{\tau}$ (or $\xi_{t}$ ), and mass $\xi_{m}$. Dimension coefficients are introduced only to give a dimensional content to physical quantities, combinations of them are universal constants of physics (20), (25), and (31). The origin of the geometric constants of the type $\pi$ and $\chi_{c}$ is related to the transition from actually formed trajectories to simplified linear trajectories with measured parameters. So, when interacting with partners, the trajectory of motion is formed as a spatial figure from mutually perpendicular $\chi_{d}$-intervals. The transition to the measured parameters occurs in two ways:

- by curving the outer outlines, which turns the initial figure also into a spatial figure in the form of a torus;

- representation of the trajectory in a linear format, while the large radius of the torus is considered as the distance of interaction, while the small radius remains unobservable even within the framework of quantum mechanics. Hence it follows that the formation of a real trajectory requires more $\varepsilon$-in- 
tervals than is assumed in calculations with measured parameters. A decrease in the number of $\mathcal{\varepsilon}$-acts due to these operations is taken into account by constant $\pi$ and $\chi_{c}$.

Constants of structural origin $H_{c}$ and $\alpha_{0}$ are characteristic criteria for the quantitative description of the phenomena of the physical world at both the subatomic and atomic-molecular levels. Starting from a certain hierarchical level, the constant $\alpha_{0}$ becomes the initial value to determine length, time, matter-energy, and related parameters.

The constant, the value of which is calculated by a certain set of quantum numbers, is associated with the regularities of structure formation at various hierarchical levels. As a result of the transition to measured values, the constant $\alpha_{c}=\alpha_{0} / \chi_{c}$ is transformed into a constant, the reciprocal of which is known as the fine structure constant, The presence of a relation between $H_{c}$ and $\alpha_{c}$ directly follows from the basic Equations (10)-(14). It is precisely by multiple changes $\alpha_{c}$ in the constant $H_{c}$ that energy scales are selected that characterize interactions of various natures and are the quantitative basis for the classification of elementary particles by mass.

\section{Conclusions}

The equations of motion of physical bodies are characterized by longitudinal and transverse components, the characteristic parameters of which become the basis for determining the property of matter "mass". The main states of the equations of motion $H_{c}$ and $\alpha_{c}$ are related, respectively, to the amplitude of oscillation of the initial particles of modeling the physical world and the patterns of structure formation at various hierarchical levels.

The origin of the inverse value of the fine structure constant is represented by the ratio of $\alpha_{0}$ and another constant related to the transition from a real trajectory of motion to a simplified trajectory with measurable parameters.

Using these constants, one can develop energy scales, which are a quantitative basis for describing the interactions of various classes and classifications of elementary particles by mass.

\section{Conflicts of Interest}

The author declares no conflicts of interest regarding the publication of this paper.

\section{References}

[1] Jammer, M. (1961) Concept of Mass in Classical and Modern Physics. Harvard University Press, Cambridge.

[2] Wilczek, P. (2006) Modern Physics Letters, 21, 701-712. https://doi.org/10.1142/S0217732306020135

[3] Gordon, K. (2013) The Mysteries of Mass. Scientific American, 32-39.

[4] Braibant, S., Giacomelli, G. and Spurio, M. (2009) Particles and Fundamental Interactions. An Introduction to Particle Physics. Springer Verlag, Heidelberg. 
[5] Bazarov, A., et al. (2018) Physical Review D, 98, Article ID: 054517. https://doi.org/10.1103/PhysRevD.98.054517

[6] Langacker, P. (2017) The Standard Model and Beyond. Second Edition, CRC Press, Boca Raton, 636 p. https://doi.org/10.1201/b22175

[7] Feynman, R.P. (1985) QED: The Strange Theory of Light and Matter. Princeton University Press, Princeton, 129.

[8] Kragh, H. (2003) Archive for History of Exact Sciences, 57, 395-431. https://doi.org/10.1007/s00407-002-0065-7

[9] Bedford, D. and Krumm, P. (2004) American Journal of Physics, 72, 969-970. https://doi.org/10.1119/1.1646135

[10] Kirakosyan, Kh. (2011) Structural Physics. Structural Content of the Basic Concepts and Physical Law. "Flinta", Moscow, 420 p. (In Russian)

[11] Kirakosyan, Kh. (2018) Theoretical Physics, 3, 1-27. https://doi.org/10.22606/tp.2018.31001

[12] Kirakosyan, Kh. (2019) New Horizons of Mathematical Physics, 3, 83-103. https://doi.org/10.22606/nhmp.2019.33001

[13] Kirakosyan, Kh. (2018) Theoretical Physics, 3, 33-36. https://doi.org/10.22606/tp.2018.32002

[14] CODATA Internationally Recommended 2014 Values of the Fundamental Physical Constants. https://physics.nist.gov/cuu/Constants

[15] Caurier, E., et al. (2005) Reviews of Modern Physics, 77, 427-488. https://doi.org/10.1103/RevModPhys.77.427

[16] Higgs, P.W. (1964) Physical Review Letters, 13, 508. https://doi.org/10.1103/PhysRevLett.13.508

[17] PDG (2008) Physics Letters B, 667, 1.

[18] Becker, K., Becker, M. and Schwarz, J. (2007) String Theory and M. Theory. A Modern Introduction. Cambridge Univ. Press, Cambridge.

[19] Ross, G. (1984) Grand Unified Theories. Westview Press, Boulder.

[20] Nambu, Y. (1952) Progress of Theoretical Physics, 7, 595-596. https://doi.org/10.1143/PTP.7.5.595

[21] Krasznahorkay, A.J., et al. (2016) Physical Review Letters, 116, Article ID: 042501. https://doi.org/10.1103/PhysRevLett.116.042501

[22] Krasznahorkay, A.J., et al. (2019) New Evidence Supporting the Existence of the Hypothetic X17 Particle.

[23] Barrow, J.D. (2002) The Constants of Nature from Alpha to Omega-The Numbers That Encode the Deepest Secrets of the Universe. Vintage. 